\title{
MSCTrees: a mean-shift based toolkit for cluster analysis of phylogenetic trees
}

\author{
Weixi $\mathrm{Li}^{1,2}$, Jerzy $\mathrm{W}$ Jaromczyk ${ }^{1 *}$ \\ From 10 ${ }^{\text {th }}$ Annual UT-ORNL-KBRIN Bioinformatics Summit 2011 \\ Memphis, TN, USA. 1-3 April 2011
}

\begin{abstract}
Background
Mean shift, an iterative technique for identifying the local maxima of a probability density function, has been successfully used as a clustering method in computer vision and image processing. We apply the mean shift technique to the high dimensional space of phylogeny trees. The basic idea behind this technique is to, given a set of sample points, shift each point in the direction of the gradient of the underlying density function in an iterative manner until the points concentrate at the local maxima of the density function and form natural clusters [1]. We have developed software named MSCTrees based on a variant of the mean shift method, called the adaptive mean shift [2], to perform cluster analysis on a set of multidimensional data points corresponding to phylogenetic trees.
\end{abstract}

\section{Methods}

MSCTrees has two components: a C program called ms_cluster which implements a clustering algorithm based on the adaptive mean shift method, and a Perl script called cluster_trees.pl, which converts phylogenetic trees to multidimensional data points and calls $m s_{-}$cluster to perform cluster analysis on the resulting points. The $m s$ cluster program, developed in C for optimized performance, takes a set of multidimensional data points as input, and outputs the clusters of the input points together with the cluster centers. The ms_cluster performs the following steps: 1) calculate the adaptive bandwidth for each data point using the $k$-Nearest Neighbor $(k N N)$ method; 2$)$ initialize a set of points using the values of the original data points; 3 ) shift the set of initialized points to new locations based on the mean shift vectors computed at each point; 4) repeat

'Department of Computer Science, University of Kentucky, Lexington, $\mathrm{KY}$, 40506, USA

Full list of author information is available at the end of the article step (3) until all points have converged; 5) merge points that have converged to the same locations into clusters. Four auto-optimized (and user-definable) parameters have been implemented to control the mean shift clustering process.

The cluster_trees.pl script uses the BioPerl modules to parse a set of phylogenetic trees as the input. It maps a phylogenetic tree to a multidimensional data point by calculating the pair-wise distances between the leaves of the tree as the dimensional values of the resulting point. The script produces as output clusters of phylogenetic trees resulting from the clustering of their corresponding data points.

\section{Results and conclusion}

We tested MSCTrees with a well-known gopher-louse data set, which contains two sets of phylogenetic trees (101 trees each) for 15 species of gophers and 15 species of lice, respectively. Separate cluster analyses were performed on the two sets of trees, followed by a cluster analysis on the combined tree sets. A significantly reduced number of clusters was obtained on the combined data, which suggests similarity between the two tree distributions and is consistent with the known coevolutionary relationship between gophers and lice. The pilot results demonstrate that the MSCTrees tool has strong potential for effective high dimensional cluster analysis of phylogenetic trees. We are also investigating other phylogenetic applications, such as identifying gene transfers via outlier detection.

\section{Acknowledgments}

This work was supported by NIH Research Project Grant Program (R01) from the Joint DMS/BIO/NIGMS Math/Bio Program under Grant No. 1R01GM086888-01 and the National Science Foundation under Grant No. 0814194.

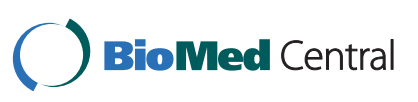

(c) 2011 Li and Jaromczyk; licensee BioMed Central Ltd. This is an open access article distributed under the terms of the Creative Commons Attribution License (http://creativecommons.org/licenses/by/2.0), which permits unrestricted use, distribution, and reproduction in any medium, provided the original work is properly cited. 


\section{Author details}

'Department of Computer Science, University of Kentucky, Lexington, KY, 40506, USA. ${ }^{2}$ Department of Biology, University of Kentucky, Lexington, KY, 40506, USA

Published: 5 August 2011

\section{References}

1. Fukunaga K, Hostetler LD: Estimation of gradient of a density-function, with applications in pattern-recognition. IEEE Transactions on Information Theory 1975, 21(1):32-40.

2. Georgescu B, Shimshoni I, Meer P: Mean shift based clustering in high dimensions: a texture classification example. Computer Vision, 2003. Proceedings. Ninth IEEE International Conference on 2003.

doi:10.1186/1471-2105-12-S7-A13

Cite this article as: Li and Jaromczyk: MSCTrees: a mean-shift based toolkit for cluster analysis of phylogenetic trees. BMC Bioinformatics 2011 12(Suppl 7):A13.

Submit your next manuscript to BioMed Central and take full advantage of:

- Convenient online submission

- Thorough peer review

- No space constraints or color figure charges

- Immediate publication on acceptance

- Inclusion in PubMed, CAS, Scopus and Google Scholar

- Research which is freely available for redistribution

Submit your manuscript at www.biomedcentral.com/submit
() Biomed Central 